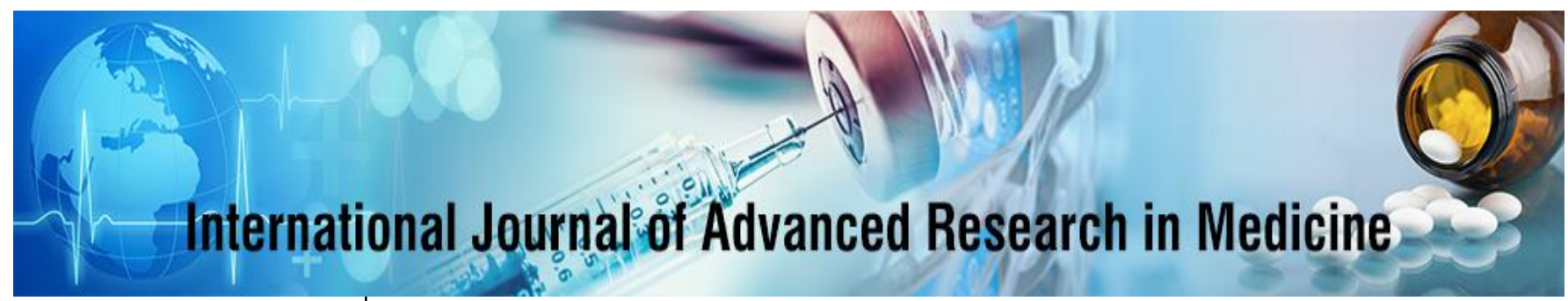

E-ISSN: 2706-9575

P-ISSN: 2706-9567

IJARM 2020; 2(2): 202-204

Received: 12-08-2020

Accepted: 17-10-2020

Dr. Arun Kumar Verma MD Medicine

Metro Hospital and Cancer Research Center, Jabalpur, Madhya Pradesh, India
Corresponding Author: Dr. Arun Kumar Verma MD Medicine

Metro Hospital and Cancer Research Center, Jabalpur, Madhya Pradesh, India

\section{Assessment of cases of epilepsy in 82 patients}

\author{
Dr. Arun Kumar Verma
}

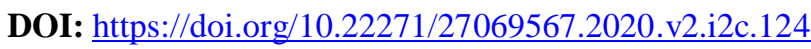

\begin{abstract}
Background: Epilepsy is a serious neurologic condition associated with stigma, psychiatric comorbidity and high economic costs. The present study was conducted to assess epilepsy in patients.

Materials \& Methods: 82 patients of epilepsy of both genders were included. A thorough clinical examination was performed and CT scan, magnetic resonance imaging (MRI) and electroencephalography was done.

Results: Out of 82 patients, males were 40 and females were 42. Common findings were focal seizures in $40 \%$, generalized tonic clonic seizures in $25 \%$, impaired consciousness in $20 \%$ and mu liple seizures in $15 \%$. The difference was significant $(\mathrm{P}<0.05)$. Common etiology was post traumatic in $22 \%$, vascular in $45 \%$, degenerative in $23 \%$ and non- identified in $10 \%$. The difference was significant $(\mathrm{P}<$ $0.05)$.

Conclusion: Common findings were focal seizures, generalized tonic clonic seizures, impaired consciousness and multiple seizures.
\end{abstract}

Keywords: Epilepsy, Neurological, Seizures

\section{Introduction}

Seizures are one of the commonest causes of transient loss of consciousness presenting to acute medical services and epileptic seizures are the commonest cause of short admissions among the neurological conditions ${ }^{[1]}$. While not all seizures are associated with lost consciousness, most requiring acute medical care will involve impaired awareness and responsiveness. Approximately $1 \%$ of the UK population are treated for epilepsy, with around 0.5 new cases per 1,000 population every year ${ }^{[1]}$. Acute medical and emergency department physicians need a good working knowledge of the management of seizures and to be aware of seizure mimics ${ }^{[2]}$.

Epilepsy is a serious neurologic condition associated with stigma, psychiatric comorbidity and high economic costs. The WHO's 2010 Global Burden of Disease study ranks epilepsy as the second most burdensome neurologic disorder worldwide in terms of disability-adjusted life years ${ }^{[3]}$. Epilepsy is a group of neurological condition characterized by seizures that are episodes that can vary from brief and nearly undetectable periods to long periods of vigorous shaking. Estimates of the prevalence and incidence of epilepsy worldwide vary considerably, likely reflecting differences in measurement and reporting, along with clinical characteristics such as etiology and seizure type ${ }^{[4]}$. Previous systematic reviews of the prevalence of epilepsy focused on specific regions ${ }^{[3]}$. Over the last decades, considerable efforts have been made to develop dedicated quality of life assessment in patients with epilepsy and to assess the impact of demographic, clinical, psychosocial factors and epilepsy-associated comorbidides on quality of life ${ }^{[5]}$. The present study was conducted to assess epilepsy in patients.

\section{Materials \& Methods}

The present study was conducted among 82 patients of epilepsy of both genders. All were informed regarding the study and written consent was obtained.

General information such as name, age, gender etc. was recorded. In all patients, causes, and symptoms were recorded. A thorough clinical examination was performed in all. Investigations such as CT scan, magnetic resonance imaging (MRI) and electroencephalography was done. Results thus obtained were subjected to statistical analysis. $\mathrm{P}$ value less than 0.05 was considered significant. 


\section{Results}

Table 1: Distribution of patients

\begin{tabular}{|c|c|c|}
\hline \multicolumn{3}{|c|}{ Total- 82 } \\
\hline Gender & Males & Females \\
\hline Number & 40 & 42 \\
\hline
\end{tabular}

Table I, graph I shows that out of 82 patients, males were 40 and females were 42.

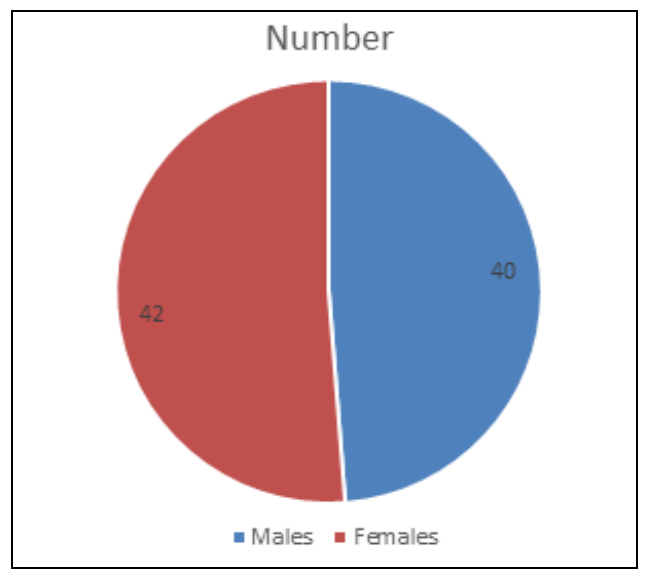

Graph I: Distribution of patients

Table II: Clinical profile in patients

\begin{tabular}{|c|c|c|}
\hline Clinical profile & Percentage & P value \\
\hline Focal seizures & $40 \%$ & \\
\cline { 1 - 2 } 0 & \multirow{2}{*}{0.03} \\
\cline { 1 - 2 } GTCS & $25 \%$ & \\
\cline { 1 - 2 } Impaired consciousness & $20 \%$ & \\
\hline Multiple seizures & $15 \%$ & \\
\hline
\end{tabular}

Table II shows that common findings were focal seizures in $40 \%$, generalized tonic clonic seizures in $25 \%$, impaired consciousness in $20 \%$ and multiple seizures in $15 \%$. The difference was significant $(\mathrm{P}<0.05)$.

Table III: Assessment of etiology in patients

\begin{tabular}{|c|c|c|}
\hline Etiology & Percentage & P value \\
\hline Post traumatic & $22 \%$ & \multirow{2}{*}{0.01} \\
\cline { 1 - 2 } Vascular & $45 \%$ & \\
\hline Degenerative & $23 \%$ & \\
\hline Non- identified & $10 \%$ & \\
\hline
\end{tabular}

Table III, graph II shows that common etiology was post traumatic in $22 \%$, vascular in $45 \%$, degenerative in $23 \%$ and non- identified in $10 \%$. The difference was significant $(\mathrm{P}<$ $0.05)$.

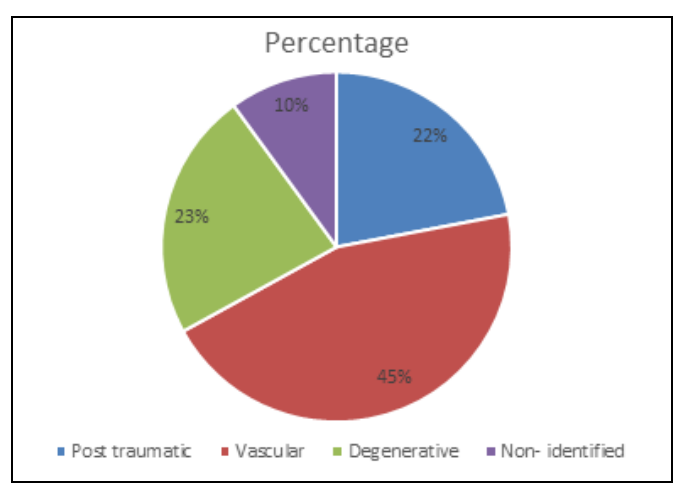

Graph II: Assessment of etiology in patients

\section{Discussion}

Transient loss of consciousness (T-LOC) is a common complaint that may present to many different specialties under various labels, including 'blackouts', 'seizure', 'blank spells', and 'falls' ${ }^{[6]}$. The term T-LOC itself is non-specific and includes all causes of self-limited loss of consciousness, regardless of mechanism. Syncope is the commonest cause of T-LOC, followed by epileptic seizures and non-epileptic seizures ${ }^{[7]}$. A thorough history including a witness account will allow a diagnosis to be made in the majority of patients. Distinguishing seizures from syncope and functional non-epileptic seizures can be difficult, but these are equally important diagnoses to make ${ }^{[8]}$. Patients with functional non-epileptic seizures are often exposed to inappropriate interventions and treatment with antiepileptic drugs. Diagnosing functional non-epileptic attack disorder and its management are covered in detail elsewhere in this issue ${ }^{[9]}$. The present study was conducted to assess epilepsy in patients.

In present study, out of 82 patients, males were 40 and females were 42 . Hauser et al. ${ }^{\mathrm{F}}$ in their study, a total of 222 studies were included (197 on prevalence, 48 on incidence). The point prevalence of active epilepsy was 6.38 per 1,000 persons $(95 \%$ confidence interval, while the lifetime prevalence was 7.60 per 1,000 persons. The annual cumulative incidence of epilepsy was 67.77 per 100,000 persons while the incidence rate was 61.44 per 100,000 person-years. The prevalence of epilepsy did not differ by age group, sex, or study quality. The active annual period prevalence, lifetime prevalence, and incidence rate of epilepsy were higher in low to middle income countries. Epilepsies of unknown etiology and those with generalized seizures had the highest prevalence.

We observed that common findings were focal seizures in $40 \%$, generalized tonic clonic seizures in $25 \%$, impaired consciousness in $20 \%$ and multiple seizures in $15 \%$. Brusturean et al. ${ }^{[11]}$ determined the impact of different clinical and demographic factor upon the quality of life. The study group was comprised by 113 patients diagnosed with epilepsy. All patients filled the quality of life in epilepsy QQLIE 31. Age was negatively correlated with almost all QQLIE items (p0.05). The employment and economic status were correlated with almost all QQLIE items.

We found that common etiology was post traumatic in $22 \%$, vascular in $45 \%$, degenerative in $23 \%$ and non- identified in $10 \%$. Harnod et al. ${ }^{[12]}$ found that cumulative incidence of epilepsy was significantly high in the migraine cohort. The aHR for developing epilepsy in the migraine cohort was 1.85. The aHR for developing epilepsy in the female migraineurs was significantly different compared with that of the non-migraine cohort and male migraineurs. The incidence of developing epilepsy was increased in patients aged 20-44 years, yielding an aHR of 2.14. The comorbidity-specific aHR for developing epilepsy associated with migraine was 2.33 in patients without any comorbidities, and 1.73 in those with comorbidities.

According to the National Institute for Health and Care Excellence (NICE) guidance, anyone with a suspected first seizure should be referred for specialist assessment and seen within 2 weeks. Most patients with a single self-terminating seizure who have made a full recovery can be managed as outpatients through local first-seizure pathways [13]. Antiepileptic medication is not indicated for a single seizure, unless investigations indicate a high risk of seizure 
recurrence. Other than in status epilepticus, starting antiepileptic drugs should ideally be a specialist decision. In the interim, the patient should be given basic safety and driving advice ${ }^{[14]}$.

\section{Conclusion}

Authors found that common findings were focal seizures, generalized tonic clonic seizures, impaired consciousness and multiple seizures.

\section{References}

1. Fernandes PT, Snape DA, Beran RG, Jacoby A. Epilepsy stigma: what do we know and where next? Epilepsy Behav 2011;22(1):55-62.

2. Berg AT, Smith SN, Frobish D, Levy SR, Testa FM, Beckerman B et al. Special education needs of children with newly diagnosed epilepsy. Dev Med Child Neurol 2005;47(11):749-53.

3. Bishop M, Boag EM. Teachers - knowledge about epilepsy and attitudes toward students with epilepsy: Results of a national survey. Epilepsy Behav 2006;8(2):397-405.

4. Abhiman, Li ML. Epilepsy in study group. J Epilepsy Clin Neurophysiol 2006;12(4):207-18.

5. Igor MN, Sayeed Khan, Akhter MS. Epilepsy in rural community of Pakistan: a description of one hundred patients, Journal of the College of Physicians and Surgeons Pakistan 2005;1:3-7.

6. Ojinnaka NC. Teachers' perception of epilepsy in Nigeria: a community-based study. Seizure 2002;11(6):386-91.

7. Souza, Dilorio C, Osborne SP, Letz R, Henry $T$, Schomer DL et al. The association of stigma with selfmanagement and perceptions of health care among adults with epilepsy. Epilepsy Behav 2003;4(3):259-67.

8. Fernandes PT, Salgado PCB, Noronha ALA, de Boer HM, Prilipko L, Sander JW, et al. Epilepsy stigma perception in an urban area of a limited-resource country. Epilepsy Behav 2007;11(1):25-32.

9. Kim MK. Marital prospects of people with epilepsy among Asians. Neurol Asia. 2007; 12:13-4.

10. Hauser WA. The prevalence and incidence of convulsive disorders in children. Epilepsia. 1994;35:S16.

11. Brusturean-Bota E, Coadă CA, Buzoianu AD, PerjuDumbravă L. Assessment of quality of life in patients with epilepsy. Human \& Veterinary Medicine. 2013;1:5(3).

12. Harnod T, Wang YC, Kao CH. High risk of developing subsequent epilepsy in young adults with migraine: a nationwide population-based cohort study in Taiwan. QJM: An International Journal of Medicine 2014;108(6):449-55.

13. Loring DW, Meador KJ, Lee GR. Determinants of quality of life in epilepsy. Epilepsy Behav 2004;5:97680.

14. Luoni C, Bisulli F, Canevini MP et al. Determinants of health-related quality of life in pharmaeoresistant epilepsy: Results from a large multicenter study of conseeutively enrolled patients using validated quantitative assessments. Epilepsia 2011;52:2181-91. 